Nervenarzt 2021 · 92:95-106

https://doi.org/10.1007/s00115-020-01031-7

Angenommen: 27. Oktober 2020

Online publiziert: 27 . November 2020

(c) Springer Medizin Verlag GmbH, ein Teil von Springer Nature 2020

\section{Hintergrund}

In Analogie zu anderen Feldern der Neurologie erlebt die Epileptologie aufgrund der intensiven Forschung einen enormen Wissenszuwachs, welcher sich auch in einer zunehmenden Individualisierung der Behandlung widerspiegeln wird. Hierunter fallen z. B. neue Erkenntnisse in der Genetik, welche zu personalisierten Therapieoptionen führen [42], die zunehmende Anzahl von Antikonvulsiva und die damit einhergehenden Kombinationsmöglichkeiten [31], die wachsende Anzahl autoimmunvermittelter Epilepsiesyndrome [7], ein besseres Verständnis über die prognostische Wertigkeit histopathologischer Befunde [22] oder die Möglichkeit, das Risiko beim Absetzen der Antikonvulsiva besser einzuschätzen [23]. Hierbei den Überblick zu bewahren, fällt Nichtexperten zwangsläufig zunehmend schwer. Hier setzen Clinical-decision-support-Systeme (CDSS) an. In letzter Zeit wurden zudem Medizin-Apps, Assistenzsysteme und telemedizinische Verfahren entwickelt, welche u. a. Onlinedatentransfer, telemedizinische Beratung, Diagnostik und Therapie ermöglichen.

Der Gesetzgeber hat kürzlich den Einsatz solcher digitaler Versorgungsansätze erleichtert [1] und durch die Bundesregierung wurde eine E-Health-Initiative zur Förderung der Digitalisierung im Gesundheitswesen aufgesetzt [8]. Auch durch die SARS-CoV-2(„severe acute respiratory syndrome coronavirus $2^{\text {“) }}$ -

\author{
Johann Philipp Zöllner ${ }^{1,2} \cdot$ Stefan Wolking ${ }^{3}$ Yvonne Weber ${ }^{3} \cdot$ Felix Rosenow $^{1,2}$ \\ 'Epilepsiezentrum Frankfurt Rhein-Main, Zentrum der Neurologie und Neurochirurgie, Goethe- \\ Universität Frankfurt, Frankfurt am Main, Deutschland \\ ${ }^{2}$ LOEWE Center for Personalized Translational Epilepsy Research (CePTER), Goethe-Universität Frankfurt, \\ Frankfurt am Main, Deutschland
}

${ }^{3}$ Epileptologie Aachen, Neurologische Uniklinik, Aachen, Deutschland

\title{
Decision-support-Systeme, Assistenzsysteme und Telemedizin in der Epileptologie
}

Pandemie ist zu erwarten, dass die Bedeutung telemedizinischer Verfahren in der Zukunft rasch zunehmen wird [43]. Dieser Beitrag gibt einen Überblick über epilepsiespezifische digitale Versorgungsansätze.

\section{Clinical-decision-support- Systeme}

\section{Definition und Bedarf}

Unter Clinical-decision-support-Systemen (CDSS) versteht man Programme, die basierend auf individuellen Patientendaten eine Entscheidungshilfe für Mediziner bieten und Informationen bündeln. Sie können klinisch tätigen Personen des Gesundheitswesens individualisierte Standards zur Diagnosestellung, Therapieentscheidungen und Sozialberatung wie auch die neuesten wissenschaftlichen Erkenntnisse für den spezifischen Fall zur Verfügung stellen. CDSS verwenden dabei Formen der Informationstechnologie und künstlichen Intelligenz zur fallbezogenen, kontextangepassten Aufarbeitung klinischer Daten, von Leitlinien sowie evidenz- und expertenbasierten Wissens. CDSS können mit anderen Tools verknüpft werden wie z.B. der automatisierten Nachbearbeitung von Rohdaten verschiedenster Zusatzdiagnostiken (zur Übersicht siehe [25, 30]; vgl. auch • Abb. 1).

Die Notwendigkeit solcher Systeme ergibt sich aus dem rasanten Wissenszuwachs über einzelne Erkrankungen, des- sen Erfassung und klinische Einordnung für Nichtexperten eine zunehmende Herausforderung darstellen kann. Die hierdurch zunehmende Entwicklung eines ärztlichen Expertenwesens führt dazu, dass Patienten häufig nicht mehr als Gesamtindividuum behandelt werden und die Gefahr besteht, dass Information zwischen den behandelnden Ärzten verloren gehen. Darüber hinaus braucht die Ausbildung von Experten viel Zeit und es wäre wünschenswert, das Wissen auch Anfängern und Nichtexperten frühzeitig zur Verfügung zu stellen. Insbesondere bei chronischen Erkrankungen basieren zudem Leitlinien auf Erkenntnissen mit zum Teil nur geringer Evidenz, sodass diese meist nur die ersten Behandlungsschritte abdecken. In der Betreuung von Patienten wäre daher oftmals frühzeitig Expertenwissen gefragt, welches häufig jedoch nur verzögert übermittelt wird. Entwicklungen, wie z. B. die langen Wartezeiten bis zur ersten prächirurgischen Evaluation [6] bei Patienten mit pharmakoresistenter Epilepsie oder die vermutlich relevante Zahl an falsch-positiv diagnostizierten Patienten [45], könnte somit entgegengewirkt werden.

In den letzten 10 bis 20 Jahren findet sich eine steigende Anzahl an Veröffentlichungen zur Entwicklung von CDSS für verschiedenste Erkrankungen. Die entwickelten Systeme beschränken sich in der einfachen Form auf die systematische Erfassung klinischer Informationen und reichen bis zu Systemen zur ausgedehnten, fallbezogenen Hilfestellung bei Dia- 


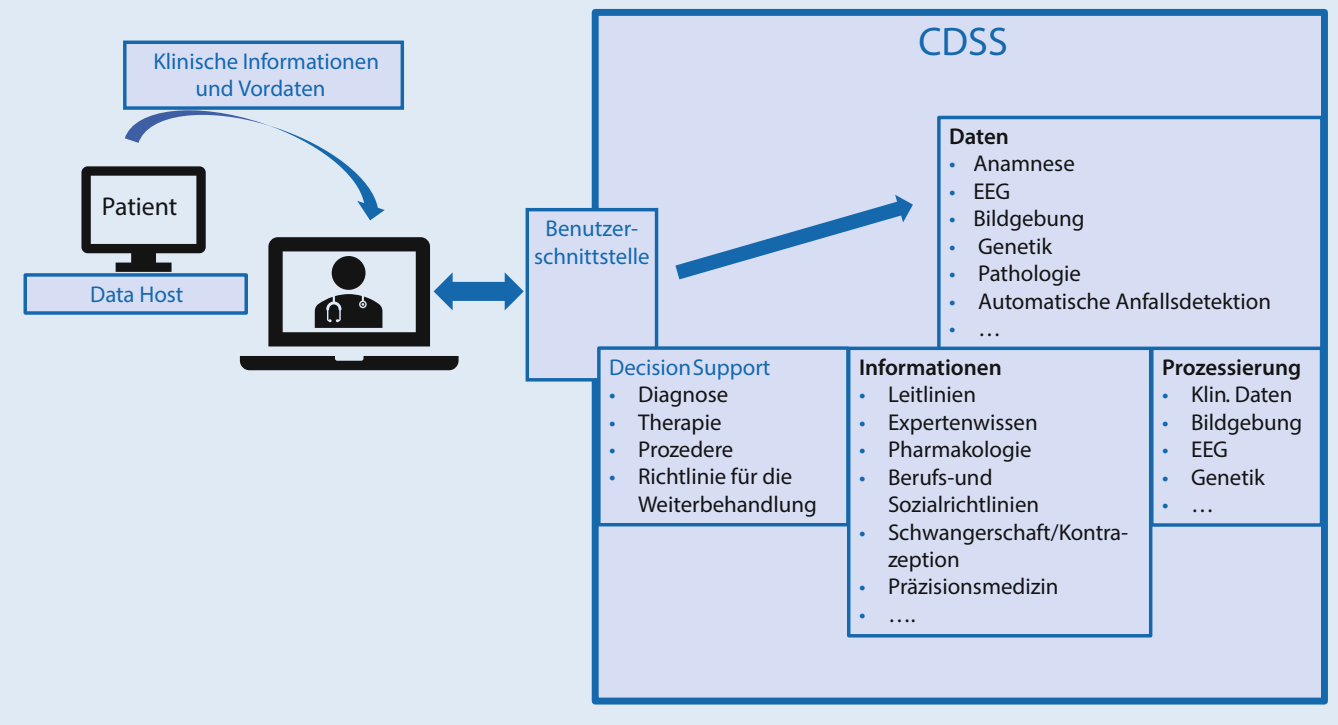

Abb. $1<$ Darstellung der Einzelbereiche eines Clinical-decision-support-Systems (CDSS) am Beispiel der Epilepsie. EEGElektroenzephalographie

gnose, medikamentösen (Indikation, Interaktion, Monitoring, Beendigung) sowie prozeduralen Therapieentscheidungen, Erkrankungsedukation und Sozialberatung. Eine Übersicht findet sich bei [30].

$\mathrm{Ob}$ ein solches System breite Anwendung findet, wird durch die Nutzbarkeit und Bedienfreundlichkeit im Alltag („usability“: z. B. selbsterklärend/ übersichtlich/schnell) sowie die Akzeptanz der Nutzer für technische Hilfestellung bestimmt. Kritikpunkte umfassen zum einen die Sorge, dass Experten ihre Expertenrolle genommen wird, zum anderen Bedenken hinsichtlich Fehlentscheidungen, Autonomieverlust sowie Abhängigkeit von Technik und ihren möglichen Fehlfunktionen.

\section{Alltagstauglichkeit und klinischer Nutzen}

In verschiedenen Studien wurde gezeigt, dass CDSS alltagstauglich sein können, Therapiemonitoring verbessert wird, medikamentöse Nebenwirkungen vermieden werden können und edukativer Mehrgewinn für den Benutzer besteht ([30, 38] sowie eigene, nicht veröffentlichte Daten). Der Mehrwert des Systems hängt aber auch von den Grundkenntnissen der Benutzer ab, d.h. Experten mit entsprechendem Vorwissen werden weniger von dem System profitieren als Allgemeinmediziner. Dementsprechend neigen Experten dazu, die Empfehlungen als aufdrängend zu empfinden und ihnen nicht $\mathrm{zu}$ folgen [25].

Gerade Personen mit Epilepsie profitieren aufgrund des chronischen Verlaufs von einer engmaschigen Dokumentation und leitliniengerechtem Handeln [28]. Aufgrund der Komplexität der Erkrankung und der psychosozialen Folgen erfordert häufig die Diagnosestellung bereits Spezialwissen [34]. Die Leitlinien umfassen aufgrund der mangelnden Studienlage mit hohem Evidenzcharakter maximal die Medikation/die Prozedur der 1. und 2. Wahl [15, 38]. Hinzu kommen bspw. die Empfehlungen zur genetischen Diagnostik, Beratung und Präzisionsmedizin [41, 44], Beachtung von Richtlinien zur Schwangerschaft und Kontrazeption [40], epilepsiechirurgische Optionen sowie die Beratung hinsichtlich der beruflichen Tätigkeit [13], Fahrtauglichkeit und weitere soziale Aspekte.

\section{》) Ein CDSS sollte eine Benutzeroberfläche für Behandelnde und Patienten bieten}

Die Studienlage für epilepsiespezifische CDSS ist bisher übersichtlich [29, 32]. Diese Systeme sind zudem nicht allumfassend, sondern beschränken sich z. B. nur auf Diagnosestellung oder Therapiefindung für bestimmte Indikationen. Wünschenswert wäre für die Epileptologie ein CDSS, das eine Benutzeroberfläche sowohl für die Behandelnden als auch Patienten bietet (• Abb. 1). Die Patienten könnten so vor der ärztlichen Vorstellung anamnestische Informationen eingeben. Datenschutzrechtlich wäre in Analogie zur elektronischen Gesundheitskarte anzustreben, dass die Daten prinzipiell den Patienten gehören und diese den Behandelnden durch ausdrückliche Zustimmung Zugriffsrechte gewähren können [11]. Letztere können nachfolgend weitere Daten eingeben wie z. B. den Anfallsbeginn, Anfallstypen etc. Diese Informationen würden mit Ergebnissen von Zusatzuntersuchungen wie Befunden aus der Elektroenzephalographie (EEG) und kraniellen Magnetresonanztomographie (cMRT) verknüpft, aber auch mit genetischen oder neuropathologischen Befunden. Diese Daten können auch mit automatisierten Nachbearbeitungsprogrammen verknüpft werden, z. B. für cMRT-Daten [10]. Auf der Basis von künstlicher Intelligenz, vorgegebenen Leitlinien und Expertenwissen wird daraus eine Diagnose gestellt, weitere Diagnostiken empfohlen und eine Therapieempfehlung vorgeschlagen. Ärzte bleiben dabei die letzte entscheidende Instanz darüber, welchen Empfehlungen in Absprache mit dem Patienten gefolgt wird. 
Hier steht eine Anzeige.

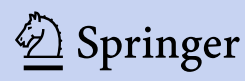


Nervenarzt 2021 · 92:95-106 https://doi.org/10.1007/s00115-020-01031-7

(c) Springer Medizin Verlag GmbH, ein Teil von Springer Nature 2020

\section{Decision-support-Systeme, Assistenzsysteme und Telemedizin in der Epileptologie}

\section{Zusammenfassung}

Hintergrund. Die wissenschaftlichen Erkenntnisse über Epilepsien und deren klinische Implikationen nehmen rasant zu. Für Nichtexperten stellt sich die zunehmende Herausforderung, den Überblick hierüber zu bewahren. Hier setzen Clinical-decisionsupport-Systeme (CDSS) an, indem sie standard- und expertengetriggertes Wissen zur Diagnostik und Therapie individualisiert und automatisiert liefern. Zudem sind Medizin-Apps und telemedizinische Verfahren zur Diagnostik und Therapie sowie Assistenzsysteme zur Anfallsdetektion bei Epilepsien verfügbar.

Ziel der Arbeit. Es soll ein Überblick über die aktuellen Entwicklungen und Anwendungsmöglichkeiten verfügbarer tele-epileptologischer Methoden gegeben werden.

Material und Methoden. Auf der Basis persönlicher Kenntnis und eines Literaturreviews werden epilepsiespezifische CDSS, MedizinApps, Assistenzsysteme sowie telemedizinische Anwendungen charakterisiert und deren klinische Einsatzmöglichkeiten dargestellt. Ergebnisse und Diskussion. Personen mit Epilepsie könnten aufgrund des chronischen Verlaufs und der Komplexität der Erkrankung und ihrer Folgen von CDSS profitieren. Es erscheint wünschenswert, dass epilepsiespezifische CDSS sowohl für die Behandelnden als auch für Patienten nutzbar werden. Apps für Menschen mit Epilepsie dienen derzeit meist der Verlaufsdokumentation von
Anfallsfrequenz, Medikamentencompliance und Nebenwirkungen. Gegenwärtige Anfallsdetektionssysteme erkennen vor allem generalisiert tonisch-klonische Anfälle (GTKA). Ein klinischer Nutzen ist noch nicht hinreichend belegt, erscheint aber wahrscheinlich, insbesondere da GTKA mit dem Risiko eines plötzlichen Todes von Epilepsiepatienten assoziiert sind und Interventionen als wirksam gelten.

Schlüsselwörter

Klinischer Nutzen · Klinische Entscheidungsfindung $\cdot$ Leitlinienwissen - GesundheitsApps - Anfallsdetektion

\section{Decision support systems, assistance systems and telemedicine in epileptology}

\section{Abstract}

Background. Scientific knowledge about epilepsies and their clinical ramifications is rapidly expanding. This becomes an even greater challenge for non-specialists to process. Clinical decision support systems (CDSS) can play an important role as an expertdriven diagnostic and therapeutic tool which gives automated and individualized advice. In addition, medical apps and telemedical procedures for diagnostics and treatment and assistance systems for seizure detection in epilepsy patients have become available. Objective. This article provides an overview on current tele-epileptological developments and the available telemedical applications.
Material and methods. Based on personal experience and a review of the literature, current epilepsy-specific CDSS, medical apps and assistance systems as well as telemedical applications are characterized and the clinical fields of application are presented.

Results and conclusion. Due to the chronic course and the complexity of the epilepsies and their sequelae, persons with epilepsy could profit from CDSS. Epilepsy-specific CDSS should be usable by medical professionals and patients themselves. Currently, medical apps for people with epilepsy are mostly used to document the clinical course, seizure frequency, medication compliance and side effects. Available seizure detection systems mainly detect generalized tonicclonic seizures (GTCS). A clinical benefit of such devices is not yet sufficiently confirmed but appears to be likely, because these seizures are specifically associated with sudden unexpected death in epilepsy patients (SUDEP) and interventions are considered to be effective.

\section{Keywords}

Clinical benefits - Clinical decision making . Guideline knowledge · Health apps · Seizure detection
Langfristig können sich allerdings die prinzipiell sehr hilfreichen CDSS nur dann in unserem Alltag etablieren, wenn die Arbeitsweise der Programme entmystifiziert wird, diese somit als $\mathrm{Me}$ dizinprodukt sicher entwickelt werden und Vertrauen für deren Routineeinsatz generiert wird. Eine Legitimierung dieser Systeme und breite Anwendung funktioniert nur, wenn einheitliche CDSS von unserem Gesundheitswesen akzeptiert und umgesetzt werden. CDSS können breit implementiert für die Patienten und ärztlichen Anwender einen erheblichen Nutzen bieten.

\section{Apps und Assistenzsysteme für Menschen mit Epilepsie}

\section{App-Typen und deren Regulation}

Unter einer „App“ - kurz für Applikation - versteht man üblicherweise eine Software, die auf mobilen Geräten eingesetzt wird [46]. Im Bereich gesundheitsbezogener Applikationen finden sich einerseits wenig regulierte Gesundheits-Apps („health apps“), die sich mit dem Ziel der Förderung von Wohlbefinden und allgemeiner Leistungsfähigkeit an die breite Bevölkerung richten [36].
Zum anderen existieren Medizin-Apps („medical apps“), die bei bestimmten Krankheiten eingesetzt werden können (• Tab. 1). Insofern diese MedizinApps unter anderem auch diagnostische, überwachende oder therapeutische Funktionen beinhalten, fallen sie unter das Medizinproduktegesetz (MPG; [9, 46]). Seit Dezember 2019 sind als Medizinprodukte eingestufte und vom Bundesinstitut für Arzneimittel und Medizinprodukte (BfARM) als „Digitale Gesundheitsanwendungen“ (DiGa) eingestufte Medizin-Apps unter bestimmten Voraussetzungen erstattungsfähig. Vor 


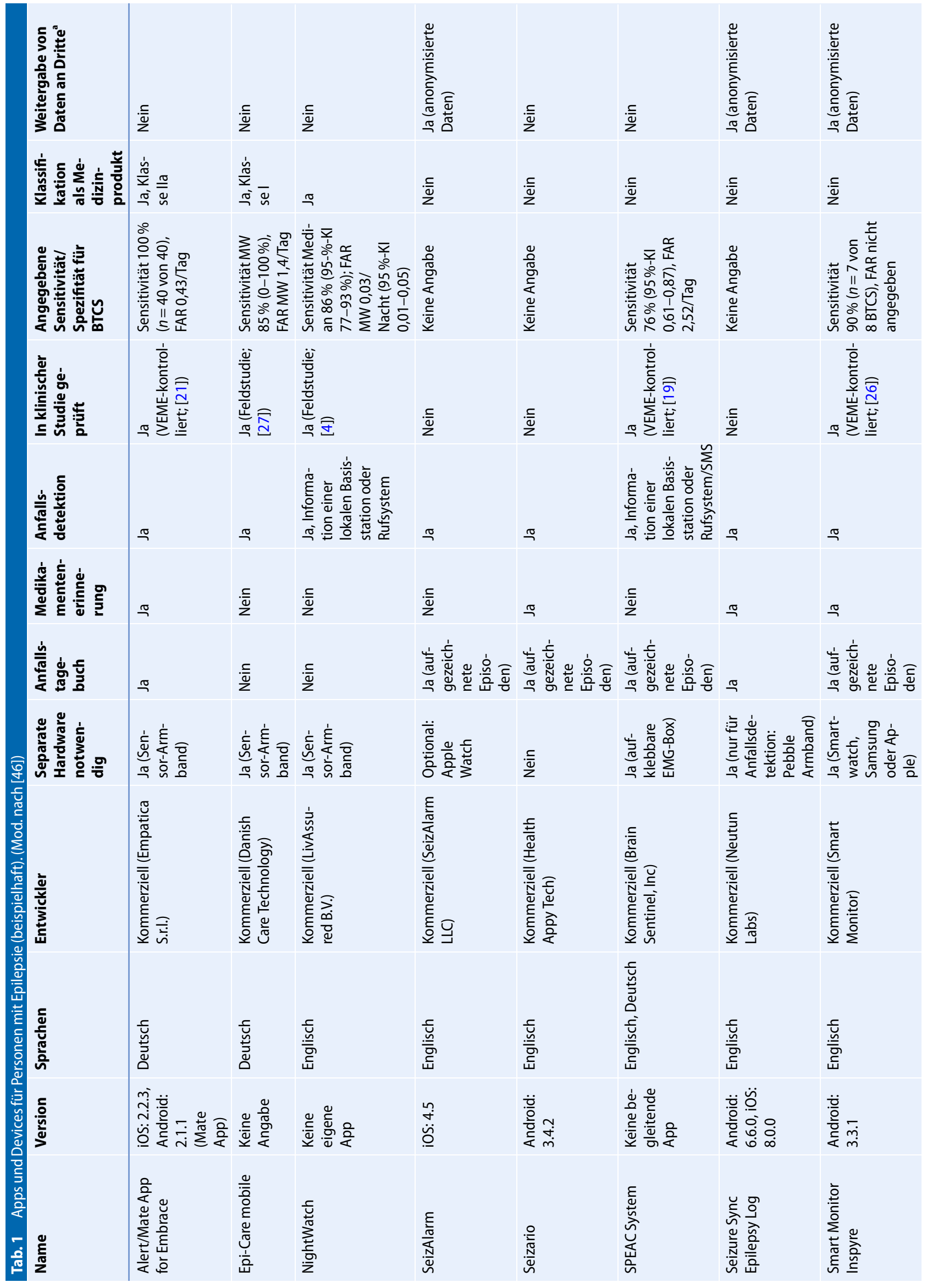




\section{Leitthema}

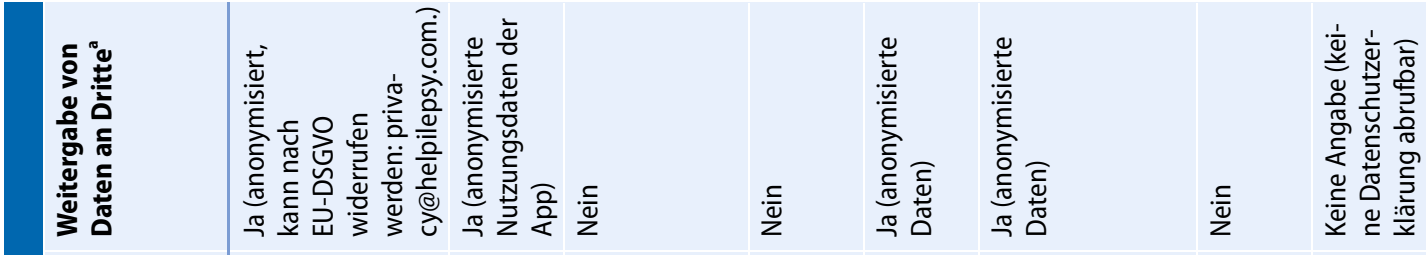

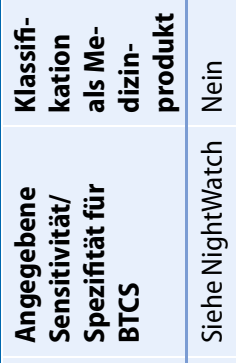

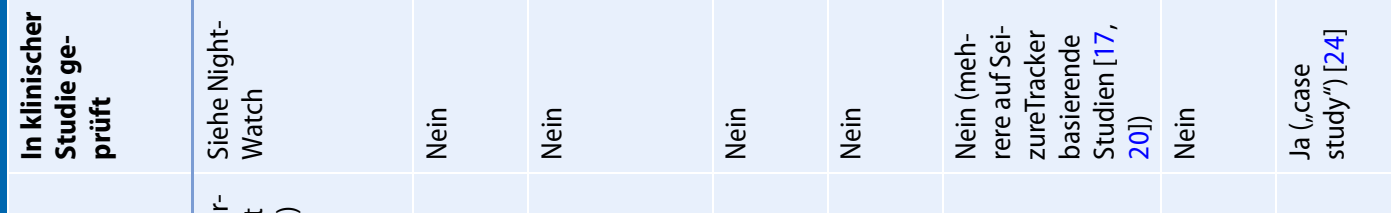

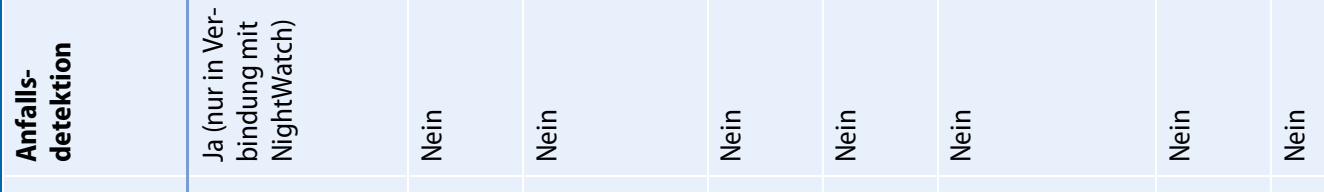

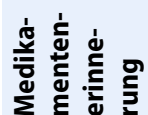

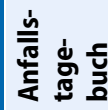

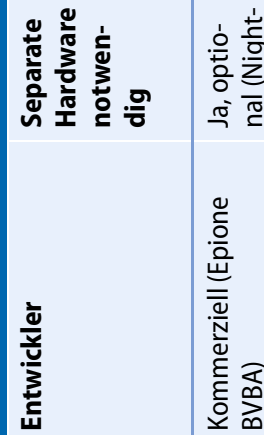

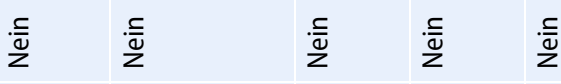

$\stackrel{\frac{c}{\underline{v}}}{\frac{\bar{v}}{2}}$

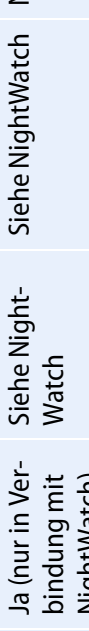

222

I

$\stackrel{2}{\pi}$

$\frac{1}{\pi} \stackrel{\frac{1}{210}}{\pi}$

끄는

$\sqrt{2} \frac{1}{2} \quad \frac{1}{6}$

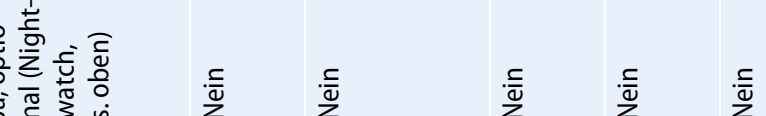

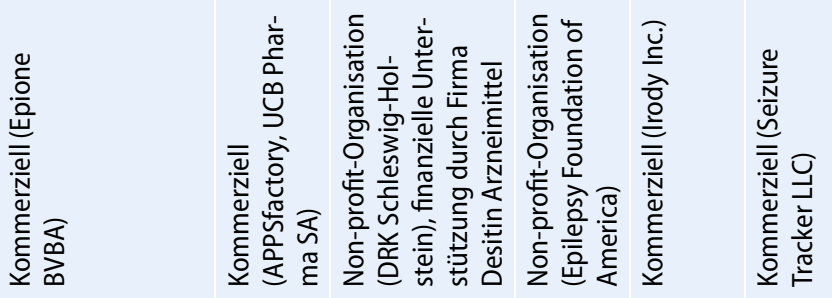

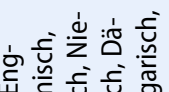

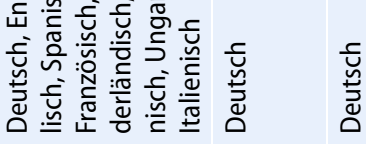

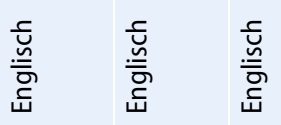

竎

i.

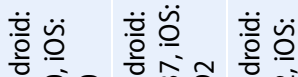

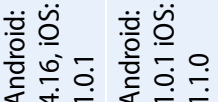

족

范定可

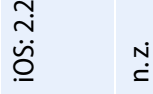

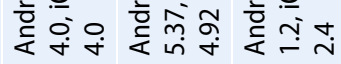

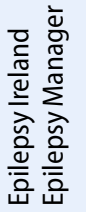

$\frac{0}{d}$

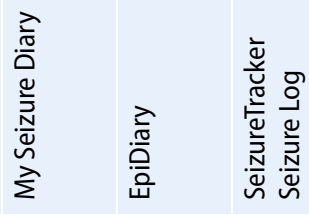

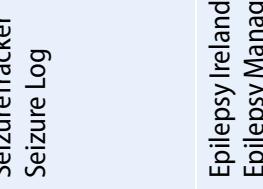

总章 
Hier steht eine Anzeige.

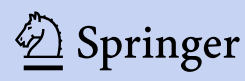


Erteilung der Erstattungsfähigkeit prüfen das BfARM und das Paul-EhrlichInstitut Versorgungseffekte, Qualität und Datenschutz der potenziellen DiGa; im Mai 2020 reichten die ersten Firmen entsprechende Anträge ein.

\section{Gesundheits-Apps für Menschen mit Epilepsie}

Die derzeit auf dem Markt befindlichen Apps für Menschen mit Epilepsie lassen sich zwei Gruppen zuordnen [46]. Die Mehrzahl der Apps sind hier den wenig regulierten Gesundheits-Apps zuzurechnen, insofern ihre Anwendung weder unmittelbare diagnostische noch therapeutische Konsequenzen haben muss. Dieser App-Typ soll vor allem die persönliche Bewältigung der Epilepsie unterstützen und bietet zu diesem Zweck Medikamentenerinnerungsalarme, Anfallskalender und teils die Möglichkeit, Anfallsvideos aufzunehmen. Als Beispiele solcher Apps seien "SeizureTracker" (www.seizuretracker.com) und „Helpilepsy" (www.helpilepsy.com) genannt. Viele dieser Gesundheits-Apps gleichen sich in ihrer Funktionalität. Leider fehlen Apps, die häufige neuropsychologische Probleme von Menschen mit Epilepsie angehen. Ebenso wird das Thema individueller Krankheitsverarbeitung oft ausgeklammert [16, 46].

\section{》) Datenschutzbestimmungen und App-Einstellungen sollten genau geprüft werden}

Es sollte zudem das Finanzierungsmodell der jeweiligen App beachtet werden: Hinter den meisten Gesundheits-Apps zum Thema Epilepsie stehen kommerzielle Anbieter oder pharmazeutische Unternehmen. Wer solche Apps empfiehlt oder anwendet sollte deshalb die Datenschutzbestimmung und App-Einstellungen genau prüfen, um z. B. die Weitergabe sensibler persönlicher Informationen zu vermeiden. Auf der anderen Seite kann das Preisgeben medizinischer Informationen in Gesundheits-Apps zu neuen Erkenntnissen über den natürlichen Verlauf der Epilepsien führen [5, 20]. Gütesiegel für Apps sind gegenwärtig wenig etabliert und in ihre Zuverlässigkeit ist oft intransparent [35], als Orientierung kann z. B. die industrieunabhängig entwickelte Bewertungsskala für medical apps „mobile app rating scale“ (MARS; [39]) eingesetzt werden.

\section{Apps zur Anfallsdetektion?}

In der zweiten Gruppe der eigentlichen "health apps" findet sich eine weit geringere Zahl an Apps. Diese sind Medizinprodukte (als solche mit CEKennzeichen) und versprechen meist eine Detektion von Bewegungsmustern oder Muskelkontraktionen, wie sie bei bestimmten epileptischen Anfällen auftreten; insofern können diese Apps derzeit nahezu ausschließlich Korrelate bilateral (generalisiert) tonisch-klonischer Anfälle erkennen. Folgerichtig sind diese Apps nicht als reine mobile Software funktionsfähig, sondern auf separate (meist externe) Sensoren angewiesen.

\section{》) Jeder Anfallsdetektor ist immer nur eine Ergänzung zur direkten Überwachung}

Zwei solche Systeme sind in Deutschland als Medizinprodukt zugelassen (Embrace; Empatica Inc., Cambridge, MA, USA [www.empatica.com] und Epi-Care mobile; Danish Care Technology ApS, Soro, Dänemark [www. danishcaretechnology.de/epicare-

mobile]). Beide benutzen externe Sensoren für Bewegungen in Form von Armbändern, um bilateral tonisch-klonische Anfälle zu detektieren [27]. Ein weiteres System (EpiHunter; epihunter, Hasselt, Belgien [www.epihunter. com]) verspricht die Detektion typisch dialeptischer Anfälle bei genetisch generalisierten Epilepsien mittels eines als Kopfband getragenen EEGs mit limitierter Elektrodenanzahl (ausschließlich Montage Fp1-F7 nach 10-20-System). Die Sensitivität dieser Systeme ist nicht absolut, worauf Patienten und Angehörige hinzuweisen sind. Zudem besteht eine gewisse Falschalarmrate. Nichtmotorische Anfälle werden von Nicht-EEGSystemen wie Embrace und Epi-Care mobile grundsätzlich nicht erkannt. Von nicht in klinischen Studien geprüften Anfallsdetektoren, die teils nur die Bewegungssensoren des Smartphones oder die einer Smartwatch nutzen, ist grundsätzlich abzuraten, da die Sicherheit, Sensitivität und Spezifität dieser Apps nicht geprüft wurde. Aber auch bei den nach MPG zugelassenen Systemen sind Patient und Angehörige darauf hinzuweisen, dass jeglicher Anfallsdetektor immer nur eine Ergänzung zur direkten Überwachung sein kann. In Einzelfällen kann solch ein System jedoch eine gewisse psychologische Absicherung bieten, wenn es mit der richtigen Zielsetzung und unter Kenntnis der medizinischen Limitationen eingesetzt wird.

Eine Übersicht aktueller Apps findet sich bei Zöllner und Strzelczyk [46], eine Liste von Systemen zur Anfallserkennung - teils in Entwicklung - findet sich unter https://www.epilepsy.com/ deviceapedia-listing-page. $\mathrm{Zu}$ beachten ist, dass die Darstellung notwendigerweise unvollständig ist. Insbesondere bei kommerziellen Anbietern unterliegt die Entwicklung von Apps und Devices einer enormen Dynamik, dabei kann die Entwicklung jederzeit eingestellt werden; auch können sich der Name oder das Finanzierungskonzept jederzeit ändern.

\section{Telemedizinische Ansätze in der Epileptologie}

\section{Definition Telemedizin und rezente Entwicklungen}

Telemedizin bedeutet die Erbringung einer medizinischen Leistung unter $\mathrm{Zu}$ hilfenahme digitaler Informations- und Kommunikationstechnologien, bei der sich die Beteiligten nicht am gleichen Ort befinden [3]. Telemedizin im Sinne der Definition der Bundesärztekammer [3] umfasst Kontakte zwischen Patient und Behandler, kann jedoch auch den Kontakt zwischen verschiedenen professionellen Behandlern (Ärzten, Pflegenden) bezeichnen [33]. Datenschutzrechtliche Aspekte der Telemedizin werden in Deutschland unter anderem sowohl in der EU-Datenschutzgrundverordnung als auch im E-Health-Gesetz sowie der 


\begin{tabular}{|c|c|c|c|c|}
\hline Name & Leitende Einrichtung & Modalität & Kooperationspartner & Kontakt \\
\hline EpilepsieNetz Hessen & $\begin{array}{l}\text { Epilepsiezentrum Frankfurt } \\
\text { Rhein-Main, Klinikum der } \\
\text { Goethe Universität Frankfurt, } \\
\text { Epilepsiezentrum Hessen, } \\
\text { Philipps-Universität Marburg }\end{array}$ & Interprofessionell & $\begin{array}{l}\text { Niedergelassene Neurologen } \\
\text { und Neuropädiater, Kliniken } \\
\text { mit neurologischer Fachabtei- } \\
\text { lung }\end{array}$ & $\begin{array}{l}\text { https://portal.epilepsienetz- } \\
\text { hessen.de } \\
\text { www.kgu.de/einrichtungen/ } \\
\text { kliniken/zentrum- } \\
\text { der-neurologie- } \\
\text { und-neurochirurgie/ } \\
\text { epilepsiezentrum-frankfurt- } \\
\text { rhein-main/ }\end{array}$ \\
\hline Tele-Epileptologie Ruhr & $\begin{array}{l}\text { Epilepsiezentrum Universi- } \\
\text { tätsklinikum Bochum }\end{array}$ & Interprofessionell & $\begin{array}{l}\text { Kliniken im Verbund der } \\
\text { Knappschaftskrankenhäu- } \\
\text { ser }\end{array}$ & $\begin{array}{l}\text { https://idp.ruhr-epileptologie. } \\
\text { de } \\
\text { www.ruhr-epileptologie.de/ } \\
\text { tele-epileptologie/ }\end{array}$ \\
\hline $\begin{array}{l}\text { Telemedizinisches Netzwerk } \\
\text { für Epilepsie in Bayern (TelEp) }\end{array}$ & $\begin{array}{l}\text { Epilepsiezentrum Universi- } \\
\text { tätsklinikum Erlangen }\end{array}$ & Interprofessionell & $\begin{array}{l}\text { Krankenhäuser und neuro- } \\
\text { logische Praxen mit einer } \\
\text { häufigen Zuweisung von } \\
\text { Epilepsiepatienten }\end{array}$ & www.telep.org/ \\
\hline $\begin{array}{l}\text { Akutneurologische Versor- } \\
\text { gung in Nordostdeutschland } \\
\text { mit telemedizinischer Unter- } \\
\text { stützung (ANNOTeM) }\end{array}$ & ANNOTeM-Konsortium & Interprofesssionell & $\begin{array}{l}\text { Kliniken mit und ohne neuro- } \\
\text { logische Fachabteilungen im } \\
\text { ANNOTeM-Verbund }\end{array}$ & www.annotem.de \\
\hline
\end{tabular}

Musterberufsordnung abgebildet (für eine Übersicht siehe [14]).

Die Entwicklungen im Bereich der Telemedizin unterliegen einem dynamischen Wandel. Mit der Lockerung des Fernbehandlungsverbotes durch den Deutschen Ärztetag im Jahr 2018 wurde die Entwicklung der Telemedizin deutlich erleichtert und mit der Verabschiedung des Digitale-VersorgungGesetzes (DVG) im November 2019 hat der Gesetzgeber die Rahmenbedingungen für die telemedizinische Behandlung von Patienten deutlich vereinfacht.

So ist durch das DVG auch eine initiale telemedizinische Kontaktaufnahme möglich geworden; auch die Abrechnung telemedizinisch erbrachter Arztleistungen ist infolge des E-Health-Gesetzes von 2015 nun einfacher möglich [2]. Infolgedessen hat eine Vielzahl kommerzieller Anbieter Lösungen entworfen, meist im Bereich der sog. „Videosprechstunden“. Teils bieten diese Lösungen auch die Möglichkeit der Übermittelung schriftlicher Befunde. Durch die Kontaktbeschränkungen im Rahmen der SARSCoV-2-Epidemie seit Anfang des Jahres 2020 hat sich die Dynamik, mit der neue telemedizinische Anwendungen auf den Markt kommen, noch einmal deutlich beschleunigt. Ein Einblick in die Nutzung von Telemedizin kann somit notwendigerweise nur ein „Schnappschuss“ sein.

\section{Tele-Epileptologie in Deutschland}

Die Anzahl an Projekten in Deutschland, die sich mit telemedizinischen Angeboten ausdrücklich für Menschen mit Epilepsie befassen, ist derzeit noch limitiert und beschränkt sich auf die interprofessionelle Kontaktebene (•Tab. 2). Inwieweit die direkte Versorgung von Menschen mit Epilepsie über nicht spezifisch epileptologische Telemedizinanwendungen (z.B. als Teil hausärztlicher Videosprechstunden) erfolgen kann, ist noch nicht erforscht. Die Deutsche Gesellschaft für Klinische Neurophysiologie und funktionelle Bildgebung (DGKN) hat im Jahr 2019 Empfehlungen für telemedizinische EEG-Untersuchungen herausgegeben [12], die Qualitätsanforderungen hinsichtlich technischer und medizinischer Aspekte von Tele-EEG-Übertragungen formulieren.

\section{EpilepsieNetz Hessen}

Seit dem Jahr 2018 besteht das von den Hessischen Ministerien für Soziales und Integration und für Wissenschaft und Kunst geförderte Telemedizinprojekt „EpilepsieNetz Hessen“ (https://portal.epilepsienetz-hessen.de), das niedergelassenen Neurologen und neurologischen Kliniken konsiliarische Unterstützung bei epileptologischen
Fragestellungen anbietet. Innerhalb des EpilepsieNetz Hessen können EEGs und jegliche Bildgebungsmodalität (MRT, Computertomographie [CT], Positronenemissionstomographie [PET]) im Originalformat überspielt werden und dann durch das Epilepsiezentrum Frankfurt Rhein-Main oder das Epilepsiezentrum Hessen in Marburg begutachtet werden, welche das EpilepsieNetz Hessen (ENH) gemeinsam organisieren. Die Übermittlung schriftlicher Befunde und der abschließenden Konsilmeinung erfolgt ebenfalls vollständig digital im Rahmen des Netzwerkes.

\section{》) Das EpilepsieNetz Hessen beurteilt explizit pädiatrische Epilepsiepatienten}

Eine Besonderheit des EpilepsieNetz Hessen ist, dass darin explizit auch die Beurteilung pädiatrischer Epilepsiepatienten integriert ist. In Zusammenarbeit mit der neuropädiatrischen Abteilung der Universitätskinderklinikin Frankfurt erfolgt ein Austausch mit anderen neuropädiatrischen und sozialpädiatrischen Zentren. In dem im Aufbau befindlichen System befinden sich bisher insgesamt sechs Kliniken und neurologische Praxen, der Anschluss weiterer acht Kliniken und Praxen erfolgt derzeit. 


\section{Tele-Epileptologie Ruhr}

Im Rahmen der „Tele-Epileptologie Ruhr“ (https://idp.ruhr-epileptologie. de) implementierte das Epilepsiezentrum des Ruhr-Klinikums Bochum die Möglichkeit zum interprofessionellen Austausch von Daten innerhalb mehrerer Kliniken des Verbundes der Knappschaftskrankenhäuser in NordrheinWestfalen. Die Tele-Epileptologie Ruhr erlaubt das Übermitteln von EEG-Daten und jeglicher Bildgebungsmodalität zum Zwecke der konsiliarischen Befundung. Gleichzeitig ist der Austausch über therapie- und diagnoserelevante Fakten per Onlinepatientenakte und integriertem Chatprogramm möglich.

\section{TelEp Erlangen}

Ein weiteres Pilotprojekt des Epilepsiezentrums in Erlangen, „TelEp“ (www. telep.org), versorgt bereits seit dem Jahr 2016 nordbayerische Kliniken mit spezialisierter epileptologischer Expertise im Rahmen von Tele-Konsilen, bei denen auch eine direkte Auswertung beim Konsilsteller lokal abgeleiteter EEGs möglich ist. Die Auswertung von über 100 Patienten erbrachte eine über 98 \%ige Zufriedenheit mit der Beratung über das TelEp-System [18, 33].

\section{ANNOTeM}

Das Konsortium „Akutneurologische Versorgung in Nordostdeutschland mit telemedizinischer Unterstützung“ (ANNOTeM) hat zum Ziel, die akutneurologische Versorgung in den beiden Flächenländern Brandenburg und Mecklenburg-Vorpommern durch ein integratives, telemedizinisch unterstütztes Behandlungskonzept $\mathrm{zu}$ verbessern [33, 37]. Das Konsortium besteht aus den klinischen Zentren Charité Universitätsmedizin Berlin, Epilepsieklinik Tabor Bernau, Unfallkrankenhaus Berlin, Universitätsmedizin Greifswald sowie regionalen Kooperationskliniken und wird unterstützt von Krankenkassen (AOK Nordost, Barmer, TK) sowie einem Telemedizinunternehmen. Durch ANNOTeM können Kliniken ohne eigene neurologische Fachabteilung ein teleneurologisches Konsil anfordern. Teil der teleneurologischen Beratung sind auch Akutkonsile in Fällen akuter unklarer Bewusstseinsstörungen und Epilepsien [33].

\section{\) Drahtlos datenübertragende EEG-Systeme können in der Notfalldiagnostik eingesetzt werden}

Darüber hinaus wird im Teilprojekt ANNOTeM-EPI der Effekt einer telemedizinisch unterstützten Differenzialdiagnose und -therapie epileptischer Anfälle auf Krankheitsverlauf und Lebensqualität untersucht. Die Klinik für Neurologie der Universität Magdeburg entwickelt und testet ein über spezielle Trockenelektroden ableitendes EEGSystem, das drahtlos funktioniert und im Rahmen von ANNOTeM für die Notfalldiagnostik eingesetzt wird. Außerdem ist es für das ambulante Monitoring neurologischer Risikopatienten außerhalb ärztlicher Praxen oder Kliniken gedacht [33]. Ziel ist es, auch in dünn besiedelten Gegenden vor Ort ein EEG-Monitoring etwa durch Pflegepersonal, Angehörige oder durch die Patienten zu ermöglichen [33].

\section{Fazit für die Praxis}

- Gerade Personen mit Epilepsie könnten aufgrund des chronischen Verlaufs und der Komplexität der Erkrankung und ihrer Folgen von Clinicaldecision-support-Systemen (CDSS) profitieren.

- Epilepsiespezifische CDSS sollten für Behandelnde und für Patienten nutzbar sein. CDSS werden sich im Alltag durchsetzen, wenn ihre Arbeitsweise entmystifiziert wird und sie als sichere Medizinprodukte entwickelt werden.

- Apps für Menschen mit Epilepsie dienen derzeit meist der Verlaufsdokumentation von Anfallsfrequenz, Medikamentencompliance und $\mathrm{Ne}$ benwirkungen.

- Aktuelle Anfallsdetektionssysteme erkennen meist motorische Anfalls- korrelate. Ihr klinischer Nutzen ist gegenwärtig nicht hinreichend belegt, erscheint aber wahrscheinlich, da generalisierte tonisch-klonische Anfälle mit einem SUDEP(,sudden unexpected death in epilepsy")-Risiko assoziiert sind und Interventionen als wirksam gelten.

- Vor dem Hintergrund des DigitaleVersorgung-Gesetzes und der SARSCoV-2(,severe acute respiratory syndrome coronavirus $2^{\prime \prime}$ )-Pandemie ist zu erwarten, dass die Bedeutung tele-epileptologischer Anwendungen zunehmen wird.

Korrespondenzadresse
PHBA. Dr. Felix Rosenow,
MHBA
Epilepsiezentrum Frankfurt
Rhein-Main, Zentrum
der Neurologie und
Neurochirurgie, Goethe-
Universität Frankfurt
Schleusenweg 2-16,
60528 Frankfurt am Main,
Deutschland
rosenow@med.uni-
frankfurt.de

\section{Einhaltung ethischer Richtlinien}

Interessenkonflikt. J.P. Zöllner erhielt Honorare für Vorträge von Desitin Arzneimittel und Eisai. S. Wolking erhielt Honorare als Redner oder Berater von den Firmen Eisai und Novartis. Y. Weber erhielt Mittel vom Ministerium für Soziales und Integration BadenWürttemberg für die Entwicklung eines EpilepsieCDSS sowie Honorare für Vorträge und Konferenzen von Desitin, UCB, Eisai, Nutricia Metabolics und Bial. F. Rosenow erhielt Beratungs- und Referentenhonorare von Arvelle Therapeutics, Desitin Arzneimittel, Eisai, GW Pharmaceuticals, Medtronic, Novartis, UCB Pharma und Unterstützung für Forschungsvorhaben der Europäischen Union, des Landes Hessen im Rahmen des LOEWE-Programmes, des Hessischen Ministeriums für Soziales und Integration und des Hessischen Ministeriums für Wissenschaft und Kunst für das EpilepsieNetz Hessen-Evaluation(ENHE)-Projekt, des Bundesministeriums für Bildung und Forschung im Rahmen des ERA-PerMed-Programmes, des Detlev-Wrobel-Fonds für Epilepsieforschung und der Deutschen Forschungsgemeinschaft.

Für diesen Beitrag wurden von den Autoren keine Studien an Menschen oder Tieren durchgeführt. Für die aufgeführten Studien gelten die jeweils dort angegebenen ethischen Richtlinien. 
Hier steht eine Anzeige.

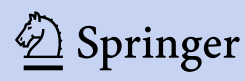




\section{Literatur}

1. http://www.bgbl.de/xaver/bgbl/start.xav? startbk=Bundesanzeiger_BGBI\&jumpTo=bgb|119 s2562.pdf.Zugegriffen: $17.0 \mathrm{kt} .2020$

2. http://www.bgbl.de/xaver/bgbl/start.xav? startbk=Bundesanzeiger_BGBI\&jumpTo=bgbl115 s2408.pdf.Zugegriffen: 17.0kt. 2020

3. https://www.bundesaerztekammer.de/fileadmin/ user_upload/downloads/pdf-Ordner/Telemedizin Telematik/Telemedizin/Telemedizinische_ Methoden_in_der_Patientenversorgung_ Begriffliche_Verortung.pdf. Zugegriffen: 23. Juli 2020

4. Arends J, Thijs RD, Gutter Tet al (2018) Multimodal nocturnal seizure detection in a residential care setting: a long-term prospective trial. Neurology 91:e2010-e2019

5. AyubN, ChiangS, Moss Retal (2020) Natural history of generalized motor seizures: a retrospective analysis. Seizure 80:109-112

6. Berg AT (2004) Understanding the delay before epilepsy surgery: who develops intractable focal epilepsy and when? CNS Spectr 9:136-144

7. Bien CG, Holtkamp M (2017) "Autoimmune Epilepsy": encephalitis with autoantibodies for epileptologists. Epilepsy Curr 17:134-141

8. https://www.bundesgesundheitsministerium.de/ e-health-initiative.html.Zugegriffen:17.0kt.2020

9. https://www.bfarm.de/DE/Medizinprodukte/ Abgrenzung/MedicalApps/_node.html. Zugegriffen:23. Juli 2020

10. Demerath T, Rubensdorfer L, Schwarzwald R et al (2020) Morphometric MRI analysis: improved detection of focal cortical dysplasia using the MP2RAGE sequence. AJNR Am J Neuroradiol 41:1009-1014

11. https://www.bfdi.bund.de/DE/Datenschutz/ Themen/Gesundheit_Soziales/Gesundheitskarte Artikel/eGK_allgemeinelnformationen.html. Zugegriffen: 17.0kt. 2020

12. https://dgkn.de/fileadmin/user_upload/pdfs/ Richtlinien/EEG/Tele-EEG-DGKN_Richtlinie.pdf. Zugegriffen: 18. Aug. 2020

13. Deutsche Gesetzliche Unfallversicherung (DGUV) (2019) DGUV Information 250-001: Berufliche Beurteilung bei Epilepsie und nach erstem epileptischen Anfall. In: Deutsche Gesetzliche Unfallversicherung (DGUV) (Hrsg) DGUV Informationen. DGUV, Berlin

14. Eickmeier $F$ (2018) Der rechtliche Rahmen für Datenschutz bei E-Health. In: Bauer C, Eickmeier $F$, Eckard M (Hrsg) E-Health: Datenschutz und Datensicherheit. Springer Gabler, Wiesbaden, S 45-52

15. Elger CE, Berkenfeld R, Beyenburg Set al (2017) S1Leitlinie Erster epileptischer Anfall und Epilepsien im Erwachsenenalter. In: Deutsche Gesellschaft für Neurologie (Hrsg) Leitlinien für Diagnostik und Therapie in der Neurologie. Thieme, Stuttgart, New York

16. Escoffery C, Mcgee R, Bidwell J et al (2018) A review of mobile apps for epilepsy self-management. Epilepsy Behav 81:62-69

17. Goldenholz DM, Rakesh K, Kapur K et al (2018) Different as night and day: Patterns of isolated seizures, clusters, and status epilepticus. Epilepsia 59:e73-e77

18. Graf W, Karampatzi F, Scibor M et al (2016) Telemedizinisches Netzwerk für Epilepsie in Bayern (TelEp). DGN-Kongress, Mannheim

19. Halford JJ, Sperling MR, Nair DR et al (2017) Detection of generalized tonic-clonic seizures using surface electromyographic monitoring Epilepsia 58:1861-1869

20. Karoly PJ, Goldenholz DM, Freestone DRetal (2018) Circadian and circaseptan rhythms in human epilepsy: a retrospective cohort study. Lancet Neurol 17:977-985

21. Lai M, Regalia G, CarboniCetal (2018) Performance assessment of Embrace, the first FDA approved smartwatch for convulsive seizure detection. Partners Against Mortality in Epilepsy (PAME), Alexandria

22. Lamberink HJ, Otte WM, Blumcke I et al (2020) Seizure outcome and use of antiepileptic drugs after epilepsy surgery according to histopathological diagnosis: a retrospective multicentre cohort study. Lancet Neurol 19:748-757

23. Lamberink HJ, Otte WM, Geerts AT et al (2017) Individualised prediction model of seizure recurrence and long-term outcomes after withdrawal of antiepileptic drugs in seizure-free patients: a systematic review and individual participant data meta-analysis. Lancet Neurol 16:523-531

24. Le Marne FA, Butler S, Beavis E et al (2018) EpApp: Development and evaluation of a smartphone/ tablet app for adolescents with epilepsy. J Clin Neurosci 50:214-220

25. Liberati EG, Ruggiero F, Galuppo L et al (2017) What hinders the uptake of computerized decision support systems in hospitals? A qualitative study and framework for implementation. Implement Sci 12:113

26. Lockman J, Fisher RS, Olson DM (2011) Detection of seizure-like movements using a wrist accelerometer. Epilepsy Behav 20:638-641

27. Meritam P, Ryvlin P, Beniczky S (2018) Userbased evaluation of applicability and usability of a wearable accelerometer device for detecting bilateral tonic-clonic seizures: a field study. Epilepsia 59(Suppl 1):48-52

28. Moura LM, Mendez DY, Jesus JD et al (2015) Association of adherence to epilepsy quality standards with seizure control. Epilepsy Res 117:35-41

29. Okazaki EM, Yao R, Sirven Jl et al (2018) Usage of EpiFinder clinical decision support in the assessment of epilepsy. Epilepsy Behav 82:140-143

30. Pearson SA, Moxey A, Robertson J et al (2009) Do computerised clinical decision support systems for prescribing change practice? A systematic review of the literature (1990-2007). BMCHealth Serv Res 9:154

31. RhoJM, WhiteHS (2018) Briefhistory of anti-seizure drug development. Epilepsia Open 3:114-119

32. Rijo R, Silva C, Pereira Letal (2014) Decision support system to diagnosis and classification of epilepsy in children. JUnivers Comput Sci 20:907-923

33. Rosenow F, Audebert HJ, Hamer HM et al (2018) Tele-EEG: Aktuelle Anwendungen, Hindernisse und technische Lösungen. Klin Neurophysiol 49:208-215

34. Scheffer IE, Berkovic S, Capovilla G et al (2017) ILAE classification of the epilepsies: position paper of the ILAE Commission for Classification and Terminology. Epilepsia 58:512-521

35. Scherenberg V (2019) Gütesiegel für Gesundheits Apps: Zwischen Vertrauen und Verunsicherung. Public Health Forum 27:225-228

36. Scherenberg V (2015) Qualitätsaspekte von Gesundheits-Apps: Wie lässt sich Qualität erkennen? Public Health Forum 23:144-146

37. Sotoodeh A, Weber JE (2019) Tele-EEG:Technikund Anwendung. Neurophysiol Labor 41:121-141
38. Standridge S, Faist R, Pestian J et al (2014) The reliability of an epilepsy treatment clinical decision support system. J Med Syst 38:119

39. Stoyanov SR, Hides L, Kavanagh DJ et al (2015) Mobile app rating scale: a new tool for assessing the quality of health mobile apps. JMIR Mhealth Uhealth 3:e27

40. Tomson T, Battino D, Bromley R et al (2019) Executive summary: management of epilepsy in pregnancy: a report from the international league against epilepsy task force on women and pregnancy. Epilepsia 60:2343-2345

41. Weber YG, Biskup S, Helbig KL et al (2017) The role of genetic testing in epilepsy diagnosis and management. Expert Rev Mol Diagn 17:739-750

42. Weber YG, Nies AT, Schwab M et al (2014) Genetic biomarkers in epilepsy. Neurotherapeutics 11:324-333

43. Willems LM, Balcik $Y$, Noda AH et al (2020) SARSCoV-2-related rapid reorganization of an epilepsy outpatient clinic from personal appointments to telemedicine services: a German single-center experience. Seizure 112:107483. https://doi.org/ 10.1016/j.yebeh.2020.107483

44. Wolking S, Schulz H, Nies AT et al (2020) Pharmacoresponse in genetic generalized epilepsy: a genome-wide association study. Pharmacogenomics 21:325-335

45. Xu Y, Nguyen D, Mohamed A etal (2016) Frequency of a false positive diagnosis of epilepsy: a systematic review of observational studies. Seizure 41:167-174

46. Zöllner JP, Strzelczyk A (2020) Nützliche Apps und Weblinks. In: Schmitt FC, Stefan H, Holtkamp M (Hrsg) Epileptische Anfälle und Epilepsien im Erwachsenenalter. Springer, Berlin 